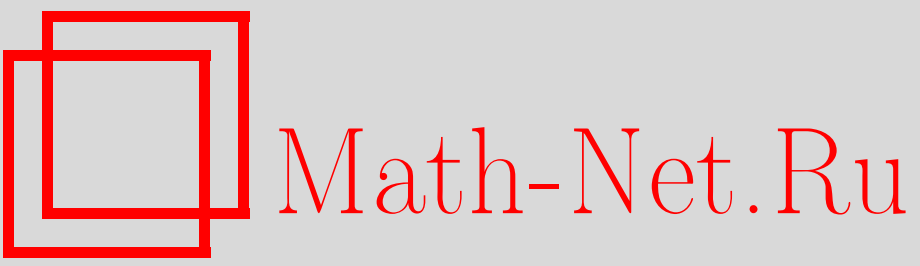

Ф. Гетце, Ю. В. Прохоров, В. В. Ульянов, Оценки для характеристических функций многочленов от асимптотически нормальных случайных величин, УМH, 1996, том 51, выпуск 2, 3-26

DOI: https://doi.org/10.4213/rm943

Использование Общероссийского математического портала Math-Net.Ru подразумевает, что вы прочитали и согласны с пользовательским соглашением

http: //www.mathnet.ru/rus/agreement

Параметры загрузки:

IP: 3.91 .87 .62

26 апреля 2023 г., $17: 21: 42$ 


\section{ОЦЕНКИ ДЛЯ ХАРАКТЕРИСТИЧЕСКИХ ФУНКЦИЙ МНОГОЧЛЕНОВ ОТ АСИМПТОТИЧЕСКИ НОРМАЛЬНЫХ СЛУЧАЙНЫХ ВЕЛИЧИН}

Ф. ГЕТце, Ю. В. ПРохоров, В. В. УЛЬЯнов

СОДЕРЖАНИЕ

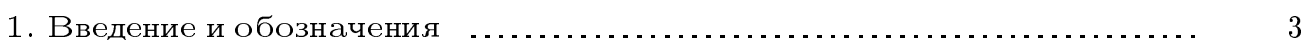

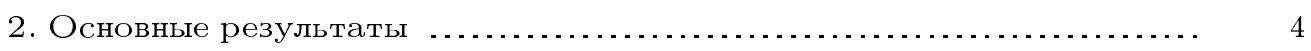

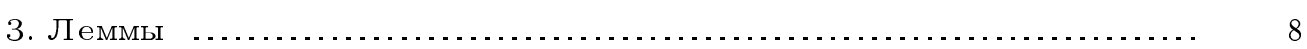

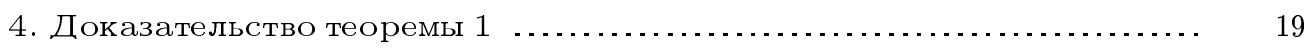

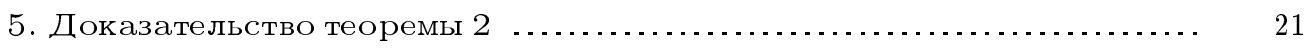

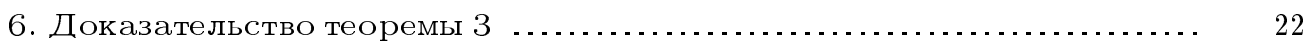

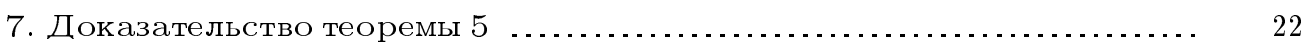

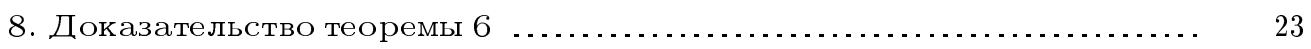

9. Доказательство теоремы 7 . . . . . . . . . . . .

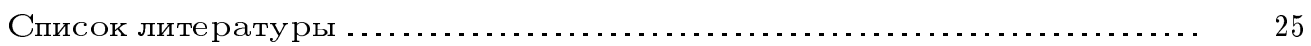

\section{1. Введение и обозначения}

Пусть через $X=\left(X^{(1)}, \ldots, X^{(k)}\right)$ обозначен случайный вектор со значениями в $k$-мерном евклидовом пространстве $\mathbb{R}^{k}$. Рассмотрим последовательность $X_{1}, X_{2}, \ldots$ независимых копий $X$. Пусть

$$
S_{n}=n^{-1 / 2}\left(X_{1}+\cdots+X_{n}\right)
$$

и $S_{n}=\left(S_{n}^{(1)}, \ldots, S_{n}^{(k)}\right)$.

В этой работе мы изучаем характеристическую функцию

$$
g_{n}(t, a)=\mathrm{E} \exp \left\{\text { it } f\left(S_{n}+a\right)\right\}, \quad a \in \mathbb{R}^{k},
$$

при больших значениях $n$ и $t$, где

$$
f(x)=\sum_{m_{1}=0}^{m} \ldots \sum_{m_{k}=0}^{m} \alpha\left(m_{1}, \ldots, m_{k}\right)\left(x^{(1)}\right)^{m_{1}} \cdots\left(x^{(k)}\right)^{m_{k}}, \quad m \geqslant 2 .
$$

Работа выполнена при поддержке Исследовательского центра 343 в Билефельдском университете, ФРГ; работа третьего автора была поддержана также Фондом им. А. Гумбольта, ФРГ.

(C) Ф. Гетце, Ю. В. Прохоров, В. В. Ульянов 1996 
Оценки для $g_{n}(t, a)$ можно получить, используя так называемое неравенство симметризации (см. [1], [2], в частности следствие 3.26 и замечание после него в [2], относяшееся к работе Вейля [3]; см. также [4]). Например, в случае $k=1$ такой подход приведет к следующему неравенству (ср. леммы 3.1, 4.1, 4.2 в [5]). Пусть

$$
f(x)=\alpha x^{m}+\alpha_{m-1} x^{m-1}+\cdots+\alpha_{0} .
$$

Для произвольной случайной величины $X$, положительных чисел $A, \varepsilon$ и любого целого $m \geqslant 2$ сушествуют положительные постоянные $c_{1}$ и $c_{2}$, зависящие от $A, \varepsilon, m$ и распределения $X$ такие, что для всех $a \in \mathbb{R}^{1}$

$$
\left|g_{n}(t, a)\right| \leqslant c_{1}\left(n^{-A}+|\alpha t|^{-1 /\left(m 2^{m}\right)}\right)
$$

при условии, что

$$
c_{2}|\alpha t| \leqslant n^{(m-1) / 2-\varepsilon} \text {. }
$$

ЗАмЕчАниЕ. Если случайньй вектор $X$ имеет дискретное распределение, то, как известно, $g_{n}(t, a)$ является почти периодической функцией (см., например, [6, с. 6] и $[7$, с. 26]) и поэтому

$$
\varlimsup_{t \rightarrow \infty}\left|g_{n}(t, a)\right|=1
$$

Следовательно, $|t|$ должно изменяться в конечном интервале, зависящем от $n$ и $f$.

Цель настояшей работы - показать, что степень $1 /\left(m 2^{m}\right)$ при $|t|^{-1}$ в (1) можно заменить большим значением в следующих двух случаях:

а) распределение $X$ имеет невырожденную дискретную компоненту (см. теоремы 1 и 2 ниже),

b) при некотором $n_{0}$ распределение суммы $S_{n_{0}}$ имеет абсолютно непрерывную компоненту (см. теоремы 3 и 4).

Кроме этого получены оценки для $\mid \mathrm{E} \exp \{$ it $f(Y+a)\} \mid$, где $Y$ - гауссовское распределение в $\mathbb{R}^{k}$ (см. теоремы 5, 6 и 7 ).

Наши доказательства опираются на неравенства для тригонометрических сумм и интегралов. В случае а) мы используем оценки типа Виноградова для тригонометрических сумм (см. [8] и лемму 6 ниже).

В случае b) применяется неравенство Виноградова для тригонометрических интегралов (см. лемму 4, гл. 2, в [9]) и его обобщения на многомерный случай (см. лемму 4 ниже). Наши уточнения оценки (1) аналогичны по форме тем улучшениями, к которым приводил метод Виноградова для тригонометрических сумм по сравнению с результатами Вейля (см. введение в [9]).

\section{2. Основные результаты}

Пусть $\mathrm{P}, \mathrm{P}_{1}, \mathrm{P}_{2}$ обозначают три вероятностные меры в $\mathbb{R}^{k}$ и $\gamma$ - положительное число, $0<\gamma \leqslant 1$. Мера $\mathrm{P}_{1}$ называется компонентой меры $\mathrm{P}$ с весом $\gamma$, если

$$
\mathbf{P}=\gamma \mathbf{P}_{1}+(1-\gamma) \mathbf{P}_{2}
$$


В теоремах 1 и 2 рассматривается одномерный случай и многочлены вида

$$
f(x)=\alpha x^{m}+\alpha_{m-1} x^{m-1}+\cdots+\alpha_{0}, \quad m \geqslant 2 .
$$

ТеОРема 1. Пусть $X_{1}, X_{2}, \ldots$ - независимые одинаково распределеннье случайные величины с распределением, имеющим компоненту $\mathrm{P}_{1}$ такую, что для некоторых чисел $a_{1}<a_{2}$ имеем

$$
\mathrm{P}_{1}\left\{a_{1}\right\}=\mathrm{P}_{1}\left\{a_{2}\right\}=\frac{1}{2}
$$

Пусть $\delta, 0<\delta \leqslant 1$, обозначает вес компоненты $\mathrm{P}_{1}$. Положсим

$$
\beta_{m}=\frac{1}{12(m-1)^{2} \ln (m-1)} .
$$

Тогда для всех $n, n>4 / \delta, u m>\widehat{c}$

$$
\begin{aligned}
\sup _{a \in \mathbb{R}^{1}}\left|\mathrm{E} \exp \left\{i t f\left(S_{n}+a\right)\right\}\right| \leqslant & 2 \exp \left(-n \delta^{2} / 8\right)+15 \widetilde{c}(m)(n \delta)^{-\beta_{m}}(\ln n)^{3 / 2} \\
& +17\left(a_{2}-a_{1}\right)^{-m /(m-1)} \delta^{-1 / 2}\left(\frac{\sqrt{n}}{|\alpha t|}\right)^{1 /(m-1)}
\end{aligned}
$$

при условии, что

$$
0<|\alpha t| \leqslant\left[\frac{\pi}{3 m \sqrt{\delta}\left(a_{2}-a_{1}\right)^{m}}(\ln n)^{-1 / 2}\right] n^{(m-1) / 2},
$$

где постоянные $\widehat{c}$ и $\widetilde{c}(m)$ те же, что и в лемме 6 ниже.

ЗАмечАниЕ. Обозначим через $L$ выражение, стояшее в квадратных скобках в правой части (4). Имеем

$$
\left(\frac{\sqrt{n}}{|\alpha t|}\right)^{1 /(m-1)} \geqslant|\alpha t|^{-m /(m-1)^{2}} L^{-1 /(m-1)^{2}} .
$$

Таким образом, последнее слагаемое в правой части (3) имеет порядок $O\left(|t|^{-m /(m-1)^{2}}\right)$ при больших $|t|$.

Теорема 2. Пусть $X_{1}, X_{2}, \ldots$ - независимые одинаково распределенные случайные величины с распределением $F$, имеющим невырожденную дискретную компоненту. Тогда для любих $\varepsilon>0$ и иелого $m \geqslant 2$ существуют абсолютние постоянные $c_{4}, c_{5}$ и постоянные $c_{2}, c_{3}$, зависящие от $F, \varepsilon$ и m такие, что для всех $n \geqslant 1$ и любого $t$, удовлетворяющего (2), справедливо неравенство

$$
\sup _{a \in \mathbb{R}^{1}}\left|\mathrm{E} \exp \left\{i t f\left(S_{n}+a\right)\right\}\right| \leqslant c_{3}\left(n^{-c_{4} /\left(m^{2} \ln m\right)}+\left(\frac{\sqrt{n}}{|\alpha t|}\right)^{c_{5} / m}\right) \text {. }
$$

Рассмотрим теперь случай непрерьвных компонент. Предположим, что случайный вектор $Y$ в $\mathbb{R}^{k}$ имеет распределение $\mathrm{P}_{Y}$ с абсолютно непрерьвной компонентой веса $\lambda$. Тогда $P_{Y}$ имеет абсолютно непрерьвную компоненту веса $\gamma, 0<\gamma<\lambda$, и такую, что плотность этой компоненты ограничена. Пусть $\gamma=\lambda / 2$. Тогда (см. [10, с. 4] или $[11, \S 16])$ распределение $\mathrm{P}_{Y} * \mathrm{P}_{Y}$ имеет компоненту веса $(\lambda / 2)^{2}$ с равномерно непрерывной плотностью, скажем, $r(x)$ на $\mathbb{R}^{k}$. Если $r\left(a_{0}\right)=r_{0}>0$ при некотором $a_{0}$, то сушествует $\eta$ такое, что при всех $x$, для которых $\left|x-a_{0}\right| \leqslant \eta$, имеем $r(x) \geqslant r_{0} / 2$. Следовательно, $\mathrm{P}_{Y} * \mathrm{P}_{Y}$ имеет компоненту, равномерно распределенную на некотором шаре. 
TEOPEMA 3. Пусmb

$$
f_{0}(x)=\left(x^{(1)}\right)^{m}+\cdots+\left(x^{(k)}\right)^{m}, \quad m \geqslant 2,
$$

и $X_{1}, X_{2}, \ldots$ обозначают последовательность независимых одинаково распределенных случайных векторов в $\mathbb{R}^{k}$. Предположим, что при некотором $n_{0}$ распределение суммы $X_{1}+\cdots+X_{n_{0}}$ имеет абсолютно непрерывную компоненту. Пусть $\eta$ обозначает действительное число такое, что распределение суммы $X_{1}+\cdots+X_{2 n_{0}}$ имеет компоненту веса $\delta$, равномерно распределенную на шаре $\left\{x:\left|x-a_{0}\right| \leqslant \eta\right\}$. Тогда для всех $n \geqslant 6 n_{0} / \delta$

$$
\sup _{a \in \mathbb{R}^{k}}\left|\mathrm{E} \exp \left\{i t f_{0}\left(S_{n}+a\right)\right\}\right| \leqslant 2 \exp \left(\frac{-n \delta^{2}}{24 n_{0}}\right)+c^{* *}(k) \eta^{-k}\left(n_{0} \delta^{-1}\right)^{k / 2}|t|^{-k / m},
$$

əде

$$
c^{* *}(k)=\left(\frac{486}{\pi}\right)^{k / 2}\left(\left(\frac{k}{2}+1\right)^{k / 2}+2^{k+1} \Gamma^{2}\left(\frac{k}{2}+1\right)\right)
$$

и Г( () есть гамма-функция.

Для компонент с дифференцируемой плотностью получен следующий результат, близкий теореме 3.

Теорема 4. Пусть $X_{1}, X_{2}, \ldots$ - последовательность независимых одинаково распределенных случайных векторов в $\mathbb{R}^{k}$ с плотностью $р$ такой, что

$$
p=\alpha p_{0}+(1-\alpha) p_{1}, \quad 0<\alpha \leqslant 1,
$$

әде $p_{0}$ непрерывно дифферениируема. Пусть

$$
V_{0}=\int_{\mathbb{R}^{k}}\left|\operatorname{grad} p_{0}(x)\right| d x .
$$

Тогда для $n \geqslant 2(k+1) / \alpha, m \geqslant 2 u t \neq 0$

$$
\sup _{a \in \mathbb{R}^{k}}\left|\mathrm{E} \exp \left\{i t f_{0}\left(S_{n}+a\right)\right\}\right| \leqslant c(k) V_{0}^{k}\left(\frac{2}{\alpha}\right)^{k / 2}|t|^{-k / m}+2 \exp \left(-\frac{\alpha^{2} n}{8}\right),
$$

где $c(k)=1.1 \cdot(17.5)^{k}$. Если $\alpha=1$, то второе слагаемое в правой части (6) мохно опустить.

Рассмотрим теперь общий многочлен вида

$$
f(x)=\sum_{m_{1}=0}^{m} \cdots \sum_{m_{k}=0}^{m} \alpha\left(m_{1}, \ldots, m_{k}\right) x_{1}^{m_{1}} \cdots x_{k}^{m_{k}}
$$

с $\alpha(0, \ldots, 0)=0$. Пусть $M$ обозначает степень многочлена $f(x)$, т.е.

$$
M=\max \left\{m_{1}+\cdots+m_{k}: \alpha\left(m_{1}, \ldots, m_{k}\right) \neq 0\right\} .
$$

Очевидно, $k m \geqslant M$. Определим

$$
\alpha_{* *}(f)=\max \left|\alpha\left(m_{1}, \ldots, m_{k}\right)\right|>0,
$$

где максимум берется по всем $m_{1}, \ldots, m_{k}$ таким, что

$$
0 \leqslant m_{1}, \ldots, m_{k} \leqslant m, \quad m_{1}+\cdots+m_{k}=M .
$$


ТеОрема 5. Пусть $Z$ - случайныи вектор в $\mathbb{R}^{k}$ с независимыми стандартньми нормальными координатами $Z^{(i)}, i=1,2, \ldots$. Для $f(x)$, определенной в $(7)$, с $M \geqslant 2$ имеем

$$
\sup _{a \in \mathbb{R}^{k}}|\mathrm{E} \exp \{i t f(Z+a)\}| \leqslant c_{6}\left|\alpha_{* *} t\right|^{-1 / m} \ln ^{c_{7}}\left(2+\left|\alpha_{* *} t\right|\right)
$$

где

$$
c_{6}=k(2 / \pi)^{1 / 2}+2^{(13 k-3 M / m) / 2}(2 \pi)^{1 / m-k / 2}(1.25 M+1)^{k-1}
$$

$u c_{7}=(3 k-M / m) / 2-1 \geqslant 0$.

Пусть теперь

$$
f_{1}(x)=x^{(1)} \cdots x^{(k)}, \quad k \geqslant 2 .
$$

Пусть $Z$, как и вьше, есть случайньй вектор в $\mathbb{R}^{k}$ с независимыми стандартными нормальными координатами. Обозначим

$$
w_{k}(t)=\mathrm{E} e^{i t f_{1}(Z)} .
$$

Легко видеть, что $w_{k}(t)$ есть смесь по $\sigma>0$ характеристических функций нормальных распределений, т.е. $\exp \left\{-t^{2} /\left(2 \sigma^{2}\right)\right\}$. Следовательно, $w_{k}(t)$ есть действительная неотрищательная четная функция, монотонно стремящаяся к нулю при $|t| \rightarrow \infty$.

ТЕОрема 6. Для любого $k \geqslant 2$ и всех $|t| \geqslant 1$ имеем

$$
l_{k}|t|^{-1} \ln ^{k-2}(|t|) \leqslant w_{k}(t) \leqslant L_{k}|t|^{-1} \ln ^{k-2}(2+|t|),
$$

əде

$$
l_{k}=2^{-1 / 2}\left(\frac{2}{(k-2)^{2} \pi e}\right)^{(k-2) / 2}
$$

$u$

$$
L_{2 m}=3^{m-1}, \quad L_{2 m+1}=2.6 \cdot 3^{m-1}, \quad m \geqslant 1 .
$$

ЗАмЕчАниЕ 1 . Применяя теорему 5 , мы получим лишњ

$$
w_{k}(t) \leqslant c(k)|t|^{-1} \ln ^{k-1}(2+|t|) .
$$

ЗАмечАниЕ 2. Для $k=2,3,4$ справедлива (см. лемму 9 и следствие леммы 11 ниже) асимптотическая формула

$$
w_{k}(t)=L_{k}^{*}|t|^{-1} \ln ^{k-2}|t|(1+o(1)) \text { при }|t| \rightarrow \infty,
$$

где $L_{k}^{*}$ - положительная постоянная. Видимо, эта формула справедлива и при $k>4$.

Рассмотрим теперь случай, когда многочлен в $\mathbb{R}^{k}$ имеет вид

$$
f_{2}(x)=\left(x^{(1)}\right)^{m_{1}} \cdots\left(x^{(k)}\right)^{m_{k}}
$$

c $m_{j} \geqslant 1, j=1,2, \ldots, k$.

Пусть, как и выше, $Z$ есть случайньй вектор в $\mathbb{R}^{k}$ с независимыми стандартными нормальными координатами $Z^{(j)}, j=1,2, \ldots, k$. 
ТеОрема 7. Предположим, что среди $m_{1}, m_{2}, \ldots, m_{k}$ в (8) существует единственное наибольшее число, скажем, $m=m_{1} u$ среди остальных $m_{j}, j=$ $2,3, \ldots, k$, существует по крайней мере одно нечетное число, скажем, $m_{2}$. Для $0<\theta<1$ обозначим

$$
z_{\theta}=\mathrm{E}\left|Z^{(1)}\right|^{-\theta}
$$

Имеем

$$
|t|^{1 / m}\left|\mathrm{E} \exp \left\{i t f_{2}(Z)\right\}\right| \uparrow c_{8} \quad n p u \quad|t| \uparrow \infty
$$

$2 \partial e$

$$
c_{8}=m^{-1} z_{1 / m}^{-1} z_{(m-1) / m} z_{m_{2} / m} \cdots z_{m_{k} / m} .
$$

\section{3. Леммы}

а) Биномиальное распределение.

ЛЕмма 1. Для любъц $p, 0 \leqslant p \leqslant 1, u \varepsilon>0$

$$
\sum_{|m-n p| \geqslant n \varepsilon}\left(\begin{array}{c}
n \\
m
\end{array}\right) p^{m}(1-p)^{n-m} \leqslant 2 e^{-n \varepsilon^{2} / 2} .
$$

Доказательство см. в [12, p. 102].

ЛЕмма 2. Для любого иелого $n \geqslant 1$

$$
\max _{0 \leqslant m \leqslant n}\left(\begin{array}{c}
n \\
m
\end{array}\right) 2^{-n} \leqslant \sqrt{\frac{2}{\pi n}} .
$$

Доказательство тривиально следует из формулы Стирлинга.

b) Равномерное распределение на единичном шаре в $\mathbb{R}^{k}$.

Пусть $U$ обозначает случайный вектор в $\mathbb{R}^{k}$ с равномерньм распределением на единичном шаре

$$
|x|^{2}:=\left(x^{(1)}\right)^{2}+\cdots+\left(x^{(k)}\right)^{2} \leqslant 1 .
$$

Его плотность, скажем, $q(x)$ дается формулой $q(x)=\Gamma(k / 2+1) \pi^{-k / 2}$, если $|x| \leqslant 1$, и $q(x)=0$ в противном случае. Характеристическая функция случайного вектора $U$ дается формулой

$$
\mathrm{E} e^{i(v, U)}=\frac{\Gamma(k / 2+1) \cdot 2^{k / 2}}{|v|^{k / 2}} J_{k / 2}(|v|),
$$

где $J_{\nu}(z)$ обозначает функцию Бесселя порядка $\nu$.

Пусть

$$
0<j_{\nu, 1}<j_{\nu, 2}<\cdots
$$

есть положительные решения уравнения $J_{\nu}(z)=0$. Нам нужен только случай $\nu=$ $k / 2, k=1,2,3, \ldots$ Известно (см., например, [13, § 15.3]), что

$$
j_{k / 2,1}>\sqrt{\frac{k}{2}\left(\frac{k}{2}+2\right)}>\frac{k}{2} .
$$


Лемма 3. Для $k=1,2,3, \ldots$

$$
\left|\mathrm{E} e^{i(v, U)}\right| \leqslant \exp \left\{-\frac{|v|^{2}}{2(k+2)}\right\}, \quad \text { ecлu }|v| \leqslant j_{k / 2,1},
$$

$u \partial \Omega я|v|>j_{k / 2,1}$

$$
\left|\mathrm{E} e^{i(v, U)}\right| \leqslant 0.75
$$

Кроме того,

$$
\int_{\mathbb{R}^{k}}\left|\mathrm{E} e^{i(v, U)}\right|^{2} d v \leqslant 2^{k} \pi^{k / 2} \Gamma\left(\frac{k}{2}+1\right) .
$$

ДОкАЗАТЕЛЬСтво. Имеем (см. [13, $§ 15.41$ и $\S 15.51])$ для всех комплексных $z$

$$
J_{\nu}(z)=\frac{(z / 2)^{\nu}}{\Gamma(\nu+1)} \prod_{l=1}^{\infty}\left(1-\frac{z^{2}}{j_{\nu, l}^{2}}\right) .
$$

Следовательно, для любого $v \in \mathbb{R}^{k}$

$$
\mathrm{E} e^{i(v, U)}=\prod_{l=1}^{\infty}\left(1-|v|^{2} j_{k / 2, l}^{-2}\right)
$$

И

$$
\sum_{l=1}^{\infty} j_{k / 2, l}^{-2}=\frac{1}{2(k+2)}
$$

Отсюда и из неравенства $1-z \leqslant e^{-z}$ вытекает (9).

Для $|v| \geqslant k / 2 \geqslant 1 / 2$

$$
\left|\mathrm{E} e^{i(v, U)}\right| \leqslant \frac{\Gamma(k / 2+1) 2^{k / 2}}{|v|^{k / 2}} \sqrt{\frac{2}{\pi|v|}}\left(1-\frac{k^{2}}{4|v|^{2}}\right)^{-1 / 4}:=G_{k}(|v|)
$$

(см. [13, $\S 13.74])$. Для $|v| \geqslant j_{k / 2,1}$ правая часть (13) не превосходит

$$
G_{k}(\sqrt{(k / 2)(k / 2+2)}) .
$$

Применяя формулу Стирлинга к $\Gamma(k / 2+1)=(k / 2) \Gamma(k / 2)$, получим

$$
G_{k}\left(\sqrt{\left(\frac{k}{2}\right)\left(\frac{k}{2}+2\right)}\right) \leqslant 2 e^{-1+3 /(k+4)+1 /(6 k)}\left(\frac{2}{e}\right)^{k / 2}\left(\frac{k}{4}+1\right)^{1 / 4} .
$$

При возрастании $k \geqslant 1$ правая часть (14) убывает, и для $k=4$ она равна

$$
e^{10 / 24} \cdot(2 / e)^{3} \cdot 2^{1 / 4}<0.75
$$

Для $k=2$ или 3 имеем $j_{1,1}=3.83171 \ldots$ и $j_{3 / 2,1}=4.493409 \ldots$ (см. [14]).

Поэтому $G_{2}\left(j_{1,1}\right)=0.21654 \ldots$ и $G_{3}\left(j_{3 / 2,1}\right)=0.15307 \ldots$ Далее, для $k=1$ имеем $j_{1 / 2,1}=\pi$ и для $|v| \geqslant \pi$

Итак, (10) доказано.

$$
\left|\mathrm{E} e^{i(v, U)}\right|=\left|\frac{\sin v}{v}\right| \leqslant \frac{1}{\pi}=0.31830 \ldots
$$

Рассмотрим теперь интеграл в (11). Имеем

$$
\frac{1}{(2 \pi)^{k}} \int_{\mathbb{R}^{k}}\left|\mathrm{E} e^{i(v, U)}\right|^{2} d v=q * q(0) \leqslant \sup _{x} q(x)=\Gamma\left(\frac{k}{2}+1\right) \pi^{-k / 2},
$$

что эквивалентно (11). Тем самым доказательство леммы 3 завершено. 
СлЕДСТВИЕ лЕммы 3. Для всех иелых $n \geqslant 2 u r, 2 \leqslant r \leqslant n$, имеем

$$
\int_{\mathbb{R}^{k}}\left|\mathrm{E} e^{i(v, U / \sqrt{n})}\right|^{r} d v \leqslant c^{*}(k)\left(\frac{n}{r}\right)^{k / 2},
$$

$2 \partial e$

$$
c^{*}(k)=2^{k} \pi^{k / 2}\left(\left(\frac{k}{2}+1\right)^{k / 2}+2^{k+1} \Gamma^{2}\left(\frac{k}{2}+1\right)\right) .
$$

ДоКАЗАТЕЛЬСТво. Интеграл в левой части (15) равен

$$
\begin{array}{r}
n^{k / 2} \int_{\mathbb{R}^{k}}\left|\mathrm{E} e^{i(v, U)}\right|^{r} d v=n^{k / 2}\left(\int_{|v|<j_{k / 2,1}}+\int_{|v| \geqslant j_{k / 2,1}}\right)\left|\mathrm{E} e^{i(v, U)}\right|^{r} d v \\
\leqslant\left(\frac{n}{r}\right)^{k / 2}(2 \pi)^{k / 2}(k+2)^{k / 2}+n^{k / 2}(0.75)^{r-2} 2^{k} \pi^{k / 2} \Gamma\left(\frac{k}{2}+1\right) .
\end{array}
$$

Заметим, что

$$
\max _{r>0} r^{k / 2}(0.75)^{r}<2^{k} \Gamma\left(\frac{k}{2}+1\right) .
$$

Тогда (15) вытекает из (16) и (17).

с) Неравенства для вероятностей больших уклонений.

Нам нужны простые верхние оценки для $\mathrm{P}\left(\left|S_{n}^{\prime}\right|>x\right)$ и $\mathrm{P}(|Y|>x)$, где $S_{n}^{\prime}=$ $n^{-1 / 2}\left(U_{1}+\cdots+U_{n}\right)$ и $U_{1}, \ldots, U_{n}-$ последовательность независимых одинаково распределенных случайных векторов, имеющих равномерное распределение на единичном шаре, т.е. $U_{n} \stackrel{\mathrm{d}}{=} U$ и $Y$ имеет гауссовское распределение с нулевым средним и невырожденной ковариационной матрицей $\Lambda=\left(\lambda_{j, l}\right), 1 \leqslant j, l \leqslant k$. Здесь и всюду ниже запись $X \stackrel{\mathrm{d}}{=} Z$ означает, что случайные величины $X$ и $Z$ одинаково распределены.

Заметим, что аналогично (12) получаем

$$
\mathrm{E} \exp (v, U)=\prod_{l=1}^{\infty}\left(1+|v|^{2} j_{k / 2, l}^{-2}\right) \leqslant \exp \left(\frac{|v|^{2}}{2(k+2)}\right)
$$

Имеем (см. [15])

$$
\begin{aligned}
\mathrm{P}\left(\left|S_{n}^{\prime}\right|>x\right) & \leqslant e^{-x^{2} / 2} \mathrm{E} \exp \left(\left|S_{n}^{\prime}\right|^{2} / 2\right) \\
& =e^{-x^{2} / 2} \mathrm{E}\left(\frac{1}{(2 \pi)^{k / 2}} \int_{\mathbb{R}^{k}} e^{\left(S_{n}^{\prime}, u\right)} e^{-|u|^{2} / 2} d u\right) \\
& \leqslant e^{-x^{2} / 2} \frac{1}{(2 \pi)^{k / 2}} \int_{\mathbb{R}^{k}} e^{|u|^{2} /(2(k+2))-|u|^{2} / 2} d u \\
& =e^{-x^{2} / 2}\left(1-\frac{1}{k+2}\right)^{-k / 2} .
\end{aligned}
$$

Второй сомножитель является убывающей функцией от $k$. Поэтому для всех $x \geqslant 0$ имеем

$$
\mathrm{P}\left(\left|S_{n}^{\prime}\right|>x\right)<e^{1 / 2-x^{2} / 2}
$$


МНОГОЧЛЕНЫ ОТ АСИМПТОТИЧЕСКИ НОРМАЛЬНЫХ СЛУЧАЙНЫХ ВЕЛИЧИН 11

Нам будет достаточно этой грубой оценки.

Для $\mathrm{P}(|Y|>x)$ имеем

$$
\mathrm{P}(|Y|>r \sigma) \leqslant 2 \exp \left(-r^{2} / 2\right)
$$

где $\sigma^{2}=\operatorname{tr} \Lambda=\mathrm{E}|Y|^{2}$. Эту оценку можно получить из неравенств в [16].

d) Тригонометрические интегралы и суммы.

Используем обозначения, введенные перед теоремой 5, в частности, рассмотрим многочлен $f(x)$ общего вида $(7)$.

Заметим, что для любого $a \in \mathbb{R}^{k}$ и $f_{a}(x):=f(x+a)$

$$
\alpha_{* *}\left(f_{a}\right)=\alpha_{* *}(f) .
$$

Определим дополнительно

$$
\alpha_{*}(f)=\max _{0 \leqslant m_{1}, \ldots, m_{k} \leqslant m}\left|\alpha\left(m_{1}, \ldots, m_{k}\right)\right| .
$$

Введем для $m>0, k \geqslant 1$ и $\alpha>0$

$$
\lambda(\alpha)=\min \left(1,32^{k} \alpha^{-1 / m} \ln ^{k-1}(\alpha+2)\right) .
$$

Легко видеть, что $\lambda(\alpha)$ есть невозрастающая функция.

ЛЕмма 4 (см. теорему $5, \S 3$, гл. 1 в [17]). Пусть

$$
I_{k}(f)=\int_{0}^{1} \cdots \int_{0}^{1} \exp \{2 \pi i f(x)\} d x .
$$

Тогда

$$
\left|I_{k}(f)\right| \leqslant \lambda\left(\alpha_{*}\right)
$$

СЛЕДСТВИЕ 1 ЛЕМмЫ 4. Для всех действительных $h>0 u a \in \mathbb{R}^{k}$ c $k \geqslant 2$

$$
\left|\int_{-h}^{h} \cdots \int_{-h}^{h} \exp \{i t f(x+a)\} d x\right| \leqslant(2 h)^{k} \lambda\left((2 \pi)^{-1}|t|(2 h)^{M} \alpha_{* *}(f)\right) .
$$

ЗАмЕчАниЕ 1 . Если $k>1$ и многочлен $f(x)$ может быть представлен в виде суммы многочленов, зависящих от различных блоков величин, например,

$$
\tilde{f}(x)=x^{(1)}\left(x^{(2)}\right)^{6}+\left(x^{(3)}\right)^{3}\left(x^{(4)}\right)^{2},
$$

то на самом деле можно получить больший показатель степени у $|t|^{-1}$ чем тот, что вытекает из следствия 1 , без учета множителя вида $\ln ^{k-1}(|t|+2)$. Например, для $\tilde{f}(x)$, указанной вьше, можно получить $|t|^{-1 / 2}$ вместо $|t|^{-1 / 6}$. См. также лемму 7 ниже.

ЗАМЕчАнИЕ 2. Одномерный случай рассмотрен в [9]. 
СлЕДСТвИЕ 2 лЕммы 4 . Для $k=1, m \geqslant 2, h>0$ и любого $a \in \mathbb{R}^{1}$

$$
\left|\int_{-h}^{h} \exp \{i t \widehat{f}(x+a)\} d x\right| \leqslant \frac{32 \cdot(2 \pi)^{1 / m}}{|\alpha t|^{1 / m}}<\frac{81}{|\alpha t|^{1 / m}},
$$

әде

$$
\widehat{f}(x)=\alpha x^{m}+\alpha_{m-1} x^{m-1}+\cdots+\alpha_{1} x .
$$

ЗАМЕчАнИЕ. В некоторых частных случаях абсолютную постоянную в правой части (18) можно заменить на меньшую.

Лемма 5 (ср. (18)). Пусть $m \geqslant 2 u$

$$
f(x)=\alpha x^{m}+\alpha_{1} x+\alpha_{0} .
$$

Тогда для всех действительных $a, b$ и $t \neq 0$

$$
\left|\int_{a}^{b} e^{i t f(x)} d x\right| \leqslant 6 \cdot\left(\frac{8}{3}\right)^{1 / 3}|\alpha t|^{-1 / m}<8.3204 \cdot|\alpha t|^{-1 / m} .
$$

ДоКАЗАТЕЛЬСТвО. Из леммы ван дер Корпута в случае $m=2$ имеем

$$
\left|\int_{a}^{b} e^{i t f(x)} d x\right| \leqslant \frac{8}{\sqrt{|t| \min \left|f^{\prime \prime}(x)\right|}}=\frac{8}{\sqrt{2}|\alpha t|^{1 / 2}},
$$

где минимум берется по всем $x, a \leqslant x \leqslant b$.

Рассмотрим теперь случай $m \geqslant 3$. Пусть $h>0$, положим

$$
N=m / 2, \quad \gamma=8(m(m-1)|\alpha t|)^{-1 / 2}, \quad \lambda(h)=h+\gamma h^{-(N-1)} .
$$

Легко видеть, что $\lambda(h)$ имеет единственный минимум в некоторой точке $h_{m}$ и

$$
\lambda\left(h_{m}\right)=d_{m}|\alpha t|^{-1 / m},
$$

где

Для $m \geqslant 3$ имеем

$$
d_{m}=\frac{m}{m-2}\left(\frac{16(m-2)^{2}}{m(m-1)}\right)^{1 / m}
$$

Запишем для $\theta>0$

$$
d_{m} \leqslant d_{3}=3\left(\frac{8}{3}\right)^{1 / 3}
$$

$$
I(\theta)=\int_{0}^{\theta} \exp \{i t f(x)\} d x .
$$

Имеем $|I(\theta)| \leqslant h_{m}$, если $0<\theta \leqslant h_{m}$. По лемме ван дер Корпута для $\theta>h_{m}$

$$
|I(\theta)| \leqslant h_{m}+\frac{8}{\sqrt{|t|\left|f^{\prime \prime}\left(h_{m}\right)\right|}}=\lambda\left(h_{m}\right) \leqslant d_{3}|\alpha t|^{-1 / m} .
$$

Это неравенство справедливо и для $\theta<0$. Поскольку

$$
\left|\int_{a}^{b} e^{i t f(x)} d x\right| \leqslant|I(b)|+|I(a)|,
$$

лемма 5 доказана.

Для тригонометрических сумм нам потребуется следуюшая 
Лемма 6 (см. [8, предложение $4.2, \S 4$, гл. VIII]). Пусть $P \geqslant 1$ и $Q$ есть иелые числа,

$$
S=\sum_{Q<x \leqslant Q+P} \exp \left\{2 \pi i\left(\alpha x^{m}+\alpha_{m-1} x^{m-1}+\cdots+\alpha_{0}\right)\right\},
$$

әде $\alpha, \alpha_{m-1}, \ldots, \alpha_{0}$ есть действительнье числа.

Предположим, что

$$
0<2 m P|\alpha| \leqslant 1 .
$$

Тогда существует постоянная $\widehat{c} \geqslant 3$, не зависящая от $m, P, Q, \alpha, \alpha_{m-1}, \ldots, \alpha_{0}$, такая, ито для $m \geqslant \widehat{c}$ имеем (ниже $m_{1}=m-1$ )

$$
|S| \leqslant \widetilde{c}(m) P^{1-1 /\left(6 m_{1}^{2} \ln m_{1}\right)} \ln P+2|\alpha|^{-1 / m_{1}},
$$

¿de

$$
\widetilde{c}(m)=2 e^{15 m_{1} \ln ^{2} m_{1}} .
$$

ЗАмЕчАниЕ. Утверждение леммы 6 можно найти также в первом издании книги Прахара (см. [8]), вьшедшем в 1957 году. В обоих изданиях это одна из трех теорем, включенных в раздел "Die Methode von Vinogradoff".

е) Функции от случайных векторов с абсолютно интегрируемыми характеристическими функциями.

Пусть $Y=\left(Y^{(1)}, \ldots, Y^{(k)}\right)$ - случайный вектор со значениями в $\mathbb{R}^{k}$. Обозначим $g_{Y}(v), v=\left(v^{(1)}, \ldots, v^{(k)}\right)$, характеристическую функцию $Y$. Пусть $p_{Y}(x), x=$ $\left(x^{(1)}, \ldots, x^{(k)}\right)$, обозначает плотность $Y$ (если она сушествует) и $\Lambda_{Y}-$ ковариационная матрица $Y$ (если $\left.\mathrm{E}|Y|^{2}<\infty\right)$. Предположим, что

$$
J_{Y}=\int_{\mathbb{R}^{k}}\left|g_{Y}(v)\right| d v<\infty .
$$

Напомним некоторые простые неравенства для $J_{Y}$. Во-первых, если $g_{Y}(v) \geqslant 0$, то $J_{Y}=(2 \pi)^{k} p_{Y}(0)$. Например, для гауссовского случайного вектора $Y$ имеем

$$
J_{Y}=(2 \pi)^{k / 2}(\operatorname{det} \Lambda)^{-1 / 2} .
$$

Далее, для всех $Y$ таких, что $\mathrm{E}|Y|^{2}<\infty$, получим (см. [18, §11.12])

$$
J_{Y} \geqslant \int_{Q_{Y} \leqslant 2}\left(1-\frac{1}{2} Q_{Y}(v)\right) d v=\left(\Gamma\left(\frac{k}{2}+1\right)\right)^{-1} 2^{k / 2} \pi^{k / 2}(\operatorname{det} \Lambda)^{-1 / 2},
$$

где

$$
Q_{Y}(v)=\mathrm{E}(v, Y-\mathrm{E} Y)^{2} .
$$

Для функций от случайных векторов рассмотрим сначала два частных случая.

Случай 1.

Для целого $m \geqslant 2$ положим

$$
f_{0}(x)=\left(x^{(1)}\right)^{m}+\cdots+\left(x^{(k)}\right)^{m} .
$$


ЛЕмма 7. При выполнении условия (19) имеем для любого а $\in \mathbb{R}^{k}$ и всех действительных $t \neq 0$

$$
\left|\mathrm{E} e^{i t f_{0}(Y+a)}\right| \leqslant 9^{k}(2 \pi)^{-k}\left(\int_{\mathbb{R}^{k}}\left|g_{Y}(v)\right| d v\right)|t|^{-k / m}
$$

ДокАЗАТЕльство (ср. [19]). Поскольку $\left|g_{Y}(v)\right|=\left|g_{Y+a}(v)\right|$, можем предполагать, что $a=0$. Запишем

$$
K(u)=\left\{x: \max _{1 \leqslant j \leqslant k}\left|x^{(j)}\right| \leqslant u\right\}
$$

Тогда

$$
\begin{aligned}
& \int_{K(u)} e^{i t f_{0}(x)} p_{Y}(x) d x \\
& =\int_{K(u)} e^{i t f_{0}(x)}\left\{\frac{1}{(2 \pi)^{k}} \int_{\mathbb{R}^{k}} e^{-i(v, x)} g_{Y}(v) d v\right\} d x \\
& =\frac{1}{(2 \pi)^{k}} \int_{\mathbb{R}^{k}} g_{Y}(v)\left\{\prod_{j=1}^{k} \int_{-u}^{u} \exp \left(-i v^{(j)} x^{(j)}+i t\left(x^{(j)}\right)^{m}\right) d x^{(j)}\right\} d v
\end{aligned}
$$

Применяя лемму 5, находим оценку сверху для модуля интеграла в левой части (21), которая не зависит от $u$. Устремляя $u$ к бесконечности, получим (20).

ПримеР. Если $Y$ имеет невырожденное нормальное распределение, то для любого $a \in \mathbb{R}^{k}$

$$
\left|\mathrm{E} \exp \left\{i t f_{0}(Y+a)\right\}\right| \leqslant c(k, \Lambda)|t|^{-k / m}
$$

где

$$
c(k, \Lambda)=(2 \pi)^{-k / 2} 9^{k}\left(\operatorname{det} \Lambda_{Y}\right)^{-1 / 2} .
$$

Заметим, что (22) было получено в следствии 8.1 в [20] другим методом и без указания вида постоянной $c(k, \Lambda)$.

В частности, при $k=2$ имеем

$$
\left|\mathrm{E} \exp \left\{i t\left(Y^{(1)}+a^{(1)}\right)^{m}+\left(Y^{(2)}+a^{(2)}\right)^{m}\right\}\right| \leqslant \frac{81}{2 \pi} \sigma_{1}^{-1} \sigma_{2}^{-1}\left(1-\rho^{2}\right)^{-1 / 2}|t|^{-2 / m}
$$

где $\sigma_{1}$ и $\sigma_{2}$ - стандартные отклонения $Y^{(1)}$ и $Y^{(2)}$, соответственно, и $\rho-$ их коэффициент корреляции. Положим при $m \geqslant 2$

$$
b_{m}= \begin{cases}\sqrt{2 / \pi} \Gamma(1+1 / m), & \text { если } m \text { четно, } \\ \sqrt{2 / \pi} \Gamma(1+1 / m) \cos (\pi /(2 m)), & \text { если } m \text { нечетно. }\end{cases}
$$

Ясно, что $b_{m}<\sqrt{2 / \pi}$ и $b_{m} \uparrow \sqrt{2 / \pi}$ при $m \rightarrow \infty$. 
Из неравенств для характеристических функций степеней нормальных случайных величин (см. [21]) получаем для независимых $Y^{(1)}, \ldots, Y^{(k)}$ с нулевыми средними (cp. (22))

$$
\sup _{t}|t|^{k / m}\left|\mathrm{E} \exp \left\{i t f_{0}(Y)\right\}\right|=b_{m}^{k} \sigma_{1}^{-1} \cdots \sigma_{k}^{-1},
$$

где $\sigma_{j}^{2}=\operatorname{Var} Y^{(j)}, j=\overline{1, k}$.

Случай 2.

Если $k=1, m \geqslant 2$ и

$$
f(x)=\alpha x^{m}+\alpha_{m-1} x^{m-1}+\cdots+\alpha_{0},
$$

то, применяя те же рассуждения, что и выше, к

$$
\int_{-h}^{h} \exp \{i t f(x+a)\} p_{Y}(x) d x=\int_{-h}^{h} \exp \{i t f(x+a)\}\left(\frac{1}{2 \pi} \int_{-\infty}^{\infty} e^{-i v x} g(v) d v\right) d x
$$

и (18), находим

$$
\left|\mathrm{E} e^{i t f(Y+a)}\right| \leqslant \frac{32(2 \pi)^{-1+1 / m}}{|\alpha t|^{1 / m}} \int_{-\infty}^{\infty}|g(v)| d v .
$$

В обшем случае для многочленов вида (7) можно оценивать характеристическую функцию с помощью неравенства

$$
\begin{aligned}
& \left|\mathrm{E} e^{i t f(Y+a)}\right| \leqslant \mathrm{P}\left(\max _{1 \leqslant j \leqslant k}\left|Y^{(j)}\right| \geqslant h\right) \\
& \quad+(2 h)^{k} \lambda\left((2 \pi)^{-1}|t|(2 h)^{M} \alpha_{* *}\right)(2 \pi)^{-k} \int_{\mathbb{R}^{k}}|g(v)| d v,
\end{aligned}
$$

выбирая $h$ как положительную функцию от $t$, стремящуюся к бесконечности при $|t| \rightarrow \infty$. Если первое слагаемое в правой части (23) экспоненциально убывает с ростом $h$, мы получим оценку для характеристической функции порядка $|t|^{-1 / m} \log ^{a}|t|$ с некоторым $a \geqslant 0$ при $|t| \rightarrow \infty$. Доказательство теоремы 5 проводится именно таким путем.

f) Многочлены специального вида от независимых нормальных случайных величин.

Лемма 8. Пусть случайная величина Х есть произведение трех независимых случайных величин:

$$
X=Z_{1}^{m} \cdot Z_{2} \cdot S
$$

где $m \geqslant 1$ есть челое, $Z_{1}, Z_{2}$ имеют стандартное нормальное распределение и $S>0$ таково, что

$$
\mathrm{E} S^{-1 / m}<\infty .
$$

Тогда

$$
|t|^{1 / m}\left|\mathrm{E} e^{i t X}\right| \uparrow d \quad \text { npu } \quad|t| \uparrow \infty,
$$

где

$$
d=\mathrm{E}\left|Z_{1}\right|^{-(m-1) / m} \mathrm{E} S^{-1 / m} .
$$


ДокАЗАТЕЛЬСтво. Очевидно, достаточно доказать лемму только для $t>0$. Имеeм

$$
Z_{1}^{m} Z_{2} S \stackrel{\mathrm{d}}{=}\left|Z_{1}\right|^{m} Z_{2} S
$$

и

$$
\begin{aligned}
t^{1 / m}\left|\mathrm{E} e^{i t X}\right|= & \int_{0}^{\infty} d \operatorname{Pr}(S<s) \\
& \times\left(t^{1 / m} \int_{0}^{\infty} \exp \left(-t^{2} v^{2} s^{2} / 2-v^{2 / m} / 2\right)(2 / \pi)^{1 / 2} m^{-1} v^{(1-m) / m} d v\right) \\
= & \int_{0}^{\infty} s^{-1 / m} d \operatorname{Pr}(S<s) \\
& \times\left(\int_{0}^{\infty} \exp \left(-\tau^{2} / 2-\tau^{2 / m} /\left[2(s t)^{2 / m}\right]\right) m^{-1}(2 / \pi)^{1 / 2} \tau^{(1-m) / m} d \tau\right)
\end{aligned}
$$

При фиксированном $s>0$ внутренний интеграл в последнем равенстве возрастает и стремится $\mathrm{K}$

$$
m^{-1} \mathrm{E}\left|Z_{1}\right|^{-(m-1) / m}=m^{-1} z_{(m-1) / m}
$$

при $t \uparrow \infty$.

Отсюда и из (24) вытекает лемма 8.

В леммах 9-11 ниже используем обозначения, введенные перед теоремой 6 . В доказательствах лемм появляются функции Бесселя $J_{0}, I_{0}, K_{0}, Y_{0}$. Все необходимые формулы для этих функций и числовые значения взяты из [14] и [22].

Лемма 9. Характеристическая функция случайной величины $Z^{(1)} Z^{(2)}$ равна

$$
w_{2}(t)=\left(1+t^{2}\right)^{-1 / 2},
$$

а ее плотность $p(v)$ при любом $v \neq 0$ определяется формулой

$$
p(v)=\pi^{-1} K_{0}(|v|) .
$$

ДокАЗАТЕЛЬСтво. Поскольку

$$
Z^{(1)} Z^{(2)} \stackrel{\mathrm{d}}{=} \frac{1}{2}\left(\left(Z^{(1)}\right)^{2}-\left(Z^{(2)}\right)^{2}\right),
$$

формула (25) вытекает из равенства

$$
\operatorname{Eexp}\left(i t\left(Z^{(1)}\right)^{2}\right)=(1-2 i t)^{-1 / 2} .
$$

Мы получаем (26) из формулы обрашения (см. 9.6.25 в [14]).

Лемма 10. Для $K_{0}(v)$ справедливы следующие неравенства:

a) $п р u 0<v \leqslant 1$

$$
K_{0}(v)<1.267 \ln (2 / v)
$$

b) $n p u v \geqslant 1$

$$
K_{0}(v) \leqslant e^{1-v} K_{0}(1) .
$$


МНОГОЧЛЕНЫ ОТ АСИМПТОТИЧЕСКИ НОРМАЛЬНЫХ СЛУЧАЙНЫХ ВЕЛИЧИН 17

ДокАЗАТЕльство. Оценка (27) вытекает из неравенства

$$
K_{0}(v)<\ln (2 / v) I_{0}(v), \quad 0<v \leqslant 1
$$

которое, в свою очередь, следует из 9.8.5 в [14].

Далее, $e^{v} K_{0}(v)$ есть убываюшая функция при $v>0$. Это следует из формулы (см. 9.6 .23 в [14])

$$
K_{0}(v)=\int_{1}^{\infty} e^{-v u}\left(u^{2}-1\right)^{-1 / 2} d u .
$$

Таким образом, (28) доказано.

СЛЕДСТВИЕ ЛЕММЫ 10. Имеем

$$
\frac{2}{\pi} \int_{1}^{\infty} K_{0}(v) d v<0.269
$$

$u \partial \Omega я t \geqslant 1$

$$
\frac{2}{\pi} \int_{0}^{1 / t} K_{0}(v) d v<1.614 t^{-1} \ln (2+t)
$$

ДокАЗАТЕльСтво. Неравенство немедленно следует из леммы 10 b). Для $t \geqslant 1$ находим

$$
\frac{2}{\pi} \int_{0}^{1 / t} K_{0}(v) d v=\frac{2}{\pi t} \int_{0}^{1} K_{0}(z / t) d z \leqslant \frac{2.534}{\pi t} \int_{0}^{1} \ln (2 t / z) d z<1.614 t^{-1} \ln (2+t)
$$

что и завершает доказательство следствия.

Лемма 11. Для $t>0$ выполнены соотношения

$$
w_{3}(t)=(2 \pi)^{-1 / 2} t^{-1} \exp \left(\left(4 t^{2}\right)^{-1}\right) K_{0}\left(\left(4 t^{2}\right)^{-1}\right)
$$

$u$

$$
w_{4}(t)=\pi(4 t)^{-1}\left(J_{0}^{2}\left((2 t)^{-1}\right)+Y_{0}^{2}\left((2 t)^{-1}\right)\right) .
$$

ДокАЗАТЕльСтво. Утверждения легко следуют из 2.3.6.10 в томе 1 и 2.16.3.12 в томе 2 в [22].

СЛЕДСТВИЕ ЛЕмМЫ 11. Имеем

$$
w_{3}(t)=(2 / \pi)^{1 / 2} t^{-1} \ln t \cdot(1+o(1)) \quad \text { npu } \quad t \rightarrow \infty
$$

$u$

$$
w_{4}(t)=\pi^{-1} t^{-1} \ln ^{2} t \cdot(1+o(1)) \quad \text { npu } \quad t \rightarrow \infty .
$$


ДокАЗАТЕльство. Следствие вытекает из (31), (32) и предельных соотношений (см. 9.6.13 и 9.1 .13 в [14])

$$
K_{0}(v)=-\ln v \cdot(1+o(1)) \quad \text { и } Y_{0}(v)=2 / \pi \ln v \cdot(1+o(1)) \quad \text { при } \quad v \downarrow 0 .
$$

g) Сведение обшей проблемы к частному случаю.

Будем использовать две простые леммы.

Лемма 12. Пусть $X$ и $Y$ - независимые случайные векторы со значениями в $\mathbb{R}^{k}$. Тогда для любой действительной борелевской функиии $f$ и всех действиmельных $t$

$$
\sup _{a \in \mathbb{R}^{k}}|\mathrm{E} \exp \{i t f(X+Y+a)\}| \leqslant \sup _{a \in \mathbb{R}^{k}}|\operatorname{E} \exp \{i t f(X+a)\}| \text {. }
$$

Доказательство очевидно.

Пусть теперь $X_{1}, X_{2}, \ldots$ - последовательность независимых одинаково распределенных случайных векторов в $\mathbb{R}^{k}$. Возьмем два целых числа $n_{1}, n, 1 \leqslant n_{1}<n$, и положим $n_{2}=\left[n / n_{1}\right]$,

$$
\begin{aligned}
Y_{1} & =X_{1}+\cdots+X_{n_{1}}, \\
Y_{2} & =X_{n_{1}+1}+\cdots+X_{2 n_{1}}, \\
\ldots \cdots \cdots \cdots \cdots \cdots \cdots \cdots \cdots & \ldots \ldots+X_{n_{2} n_{1}}, \\
Y_{n_{2}} & =X_{\left(n_{2}-1\right) n_{1}+1}+\cdots \cdots+X_{n} \\
R & =X_{n_{2} n_{1}+1}+\cdots+\cdots
\end{aligned}
$$

(если $n=n_{2} n_{1}, R=0$ ).

Пусть распределение $Y_{1}$ есть смесь распределений $Q_{1}$ и $Q_{2}$ с весами $\delta, 0<\delta \leqslant 1$, и $1-\delta$, соответственно.

Можно записать

$$
\begin{aligned}
\mathrm{E} \exp \{ & \text { it } \left.f\left(X_{1}+\cdots+X_{n}+a\right)\right\} \\
= & \sum_{j=0}^{n_{2}}\left(\begin{array}{c}
n_{2} \\
j
\end{array}\right) \delta^{j}(1-\delta)^{n_{2}-j} \\
& \quad \times \mathrm{E} \exp \left\{\text { it } f\left(Y_{1,1}+\cdots+Y_{1, j}+Y_{2, j+1}+\cdots+Y_{2, n_{2}}+\widehat{R}+a\right)\right\},
\end{aligned}
$$

где $Y_{1,1}, \ldots, \widehat{R}$ - независимые случайные векторы; случайные векторы $Y_{1,1}, \ldots, Y_{1, j}$ имеют распределение $Q_{1}$, а $Y_{2, j+1}, \ldots, Y_{2, n_{2}}$ имеют одно и то же распределение $Q_{2}$ и $\widehat{R} \stackrel{\mathrm{d}}{=} R$. Разделим сумму вьше на две части в соответствии со значением $j:\left|j-n_{2} \delta\right| \geqslant$ $n_{2} \delta / 2$ или $\left|j-n_{2} \delta\right|<n_{2} \delta / 2$.

Применяя леммы 1 и 12 , находим

$$
\begin{aligned}
\sup _{a \in \mathbb{R}^{k}} \mid \mathrm{E} \exp \{i t f( & \left.\left.X_{1}+\cdots+X_{n}+a\right)\right\} \mid \\
& \leqslant 2 \exp \left(-\frac{n_{2} \delta^{2}}{8}\right)+\sup _{a \in \mathbb{R}^{k}}\left|\mathrm{E} \exp \left\{i t f\left(Y_{1,1}+\cdots+Y_{1, n_{3}}+a\right)\right\}\right|,
\end{aligned}
$$

где $n_{3}=\left[n_{2} \delta / 2\right]+1$. Заменяя $f(x)$ на $f(x / \sqrt{n})$, получаем следующее утверждение. 
ЛЕмма 13. В обозначениях, введенных выше, имеем

$$
\begin{aligned}
& \sup _{a \in \mathbb{R}^{k}}\left|\mathrm{E} \exp \left\{i t f\left(S_{n}+a\right)\right\}\right| \\
& \quad \leqslant 2 \exp \left(-\frac{n_{2} \delta^{2}}{8}\right)+\sup _{a \in \mathbb{R}^{k}}\left|\mathrm{E} \exp \left\{i t f\left(\frac{Y_{1,1}+\cdots+Y_{1, n_{3}}}{\sqrt{n}}+a\right)\right\}\right| .
\end{aligned}
$$

\section{4. Доказательство теоремы 1}

Пусть

$$
f(x)=\alpha x^{m}+\alpha_{m-1} x^{m-1}+\cdots+\alpha_{0},
$$

где $\alpha \neq 0$ и $m$ - целое, $m \geqslant 2$. Пусть $\varepsilon_{1}, \varepsilon_{2}, \ldots$ - последовательность независимых одинаково распределенных случайных величин, принимающих значения 0 и 1 с вероятностями $1 / 2$.

Возьмем $n_{1}=1, n_{2}=n, n_{3}=[n \delta / 2]+1$. Положим

$$
\begin{gathered}
\Delta=\frac{a_{2}-a_{1}}{\sqrt{n}}, \quad \tau=(2 \pi)^{-1} t \Delta^{m} \\
f_{1}(x)=\Delta^{-m} f(\Delta x)=\alpha x^{m}+\Delta^{-1} \alpha_{m-1} x^{m-1}+\cdots+\Delta^{-m} \alpha_{0} .
\end{gathered}
$$

Тогда

$$
\begin{gathered}
\frac{Y_{1, j}}{\sqrt{n}} \stackrel{\mathrm{d}}{=} \frac{a_{1}}{\sqrt{n}}+\frac{a_{2}-a_{1}}{\sqrt{n}} \cdot \varepsilon_{j}=\frac{a_{1}}{\sqrt{n}}+\Delta \varepsilon_{j}, \\
t f\left(\frac{Y_{1,1}+\cdots+Y_{1, n_{3}}}{\sqrt{n}}+a\right) \stackrel{\mathrm{d}}{=} t f\left(\Delta\left(\varepsilon_{1}+\cdots+\varepsilon_{n_{3}}\right)+\frac{n_{3} a_{1}}{\sqrt{n}}+a\right) \\
=2 \pi \tau f_{1}\left(\varepsilon_{1}+\cdots+\varepsilon_{n_{3}}+a^{\prime}\right) .
\end{gathered}
$$

Таким образом,

$$
\begin{aligned}
\sup _{a \in \mathbb{R}^{k}}\left|\operatorname{Eexp}\left\{i t f\left(\frac{Y_{1,1}+\cdots+Y_{1, n_{3}}}{\sqrt{n}}+a\right)\right\}\right| \\
\leqslant \sup _{a^{\prime} \in \mathbb{R}^{k}}\left|\operatorname{E} \exp \left\{2 \pi i \tau f_{1}\left(\varepsilon_{1}+\cdots+\varepsilon_{n_{3}}+a^{\prime}\right)\right\}\right|
\end{aligned}
$$

Для упрошения записи, положим $r=n_{3}$. Имеем

$$
\mathrm{E} \exp \left\{2 \pi i \tau f_{1}\left(\varepsilon_{1}+\cdots+\varepsilon_{r}+a^{\prime}\right)\right\}=\sum_{j=0}^{r} \exp \left\{2 \pi i \tau f_{1}\left(j+a^{\prime}\right)\right\} p_{r}(j)=\widetilde{S}
$$

где

$$
p_{r}(j)=\left(\begin{array}{l}
r \\
j
\end{array}\right) 2^{-r}
$$

Вероятности $p_{r}(j)$ не убывают (не возрастают, соответственно), когда $j$ меняются от 0 до $[r / 2]+1$ (от $[r / 2]+1$ до $n$, соответственно). Далее, по лемме 2

$$
\max _{0 \leqslant j \leqslant r} p_{r}(j) \leqslant \sqrt{\frac{2}{\pi r}} .
$$


Напомним, что преобразование Абеля для любых действительных чисел $a_{l}$ и $b_{l}$ дает

$$
\sum_{1}^{N} a_{l} b_{l}=a_{N} B_{N}-\sum_{1}^{N-1} B_{l}\left(a_{l+1}-a_{l}\right),
$$

где

$$
B_{l}=b_{1}+\cdots+b_{l}, \quad l=1,2, \ldots, N .
$$

Если последовательность неотрицательных действительных чисел $a_{1}, \ldots, a_{N}$ такова, что существует $l_{0}, 1 \leqslant l_{0} \leqslant N$, при котором

$$
a_{1} \leqslant a_{2} \leqslant \cdots \leqslant a_{l_{0}}, \quad a_{N} \leqslant a_{N-1} \leqslant \cdots \leqslant a_{l_{0}},
$$

то из (36) следует неравенство

$$
\left|\sum_{1}^{N} a_{l} b_{l}\right| \leqslant 3 a_{l_{0}} \max _{j}\left|B_{j}\right| .
$$

Оценим теперь сумму $\widetilde{S}$ в $(35)$.

Положим

$$
A=[\sqrt{r \ln r}]+1
$$

Тогда по леммам 1,2 и 6 для $m \geqslant \widehat{c}$

$$
\begin{aligned}
|\widetilde{S}| & \leqslant \sum_{|j-r / 2| \geqslant A} p_{r}(j)+\left|\sum_{r / 2+A>j>r / 2-A} \exp \left\{2 \pi i \tau f_{1}\left(j+a^{\prime}\right)\right\} p_{r}(j)\right| \\
& \leqslant 2 \exp \left(-\frac{1}{2} r\left(\frac{A}{r}\right)^{2}\right)+3 \sqrt{\frac{2}{\pi r}} \max _{r / 2-A<k<r / 2+A}\left|\sum_{r / 2-A<j \leqslant k} \exp \left\{2 \pi i \tau f_{1}\left(j+a^{\prime}\right)\right\}\right| \\
& \leqslant \frac{2}{\sqrt{r}}+3 \sqrt{\frac{2}{\pi r}}\left[\widetilde{c}(m)(2 A)^{1-1 /\left(6 m_{1}^{2} \ln m_{1}\right)} \ln (2 A)+2|\alpha \tau|^{-1 / m_{1}}\right]
\end{aligned}
$$

при условии, что

$$
0<4 m A|\alpha \tau| \leqslant 1
$$

Наконец, оценим слагаемые в правой части (37). В предположениях теоремы 1

$$
r=\left[\frac{n \delta}{2}\right]+1>\frac{n \delta}{2} \geqslant 3 .
$$

Легко видеть, что

$$
\begin{aligned}
3 \sqrt{\frac{2}{\pi r}} \cdot 2|\alpha \tau|^{-1 / m_{1}} & =6 \sqrt{\frac{2}{\pi}} \sqrt{\frac{n}{r}}(2 \pi)^{1 / m_{1}}\left(a_{2}-a_{1}\right)^{-m /(m-1)}\left(\frac{\sqrt{n}}{|\alpha t|}\right)^{1 / m_{1}} \\
& <12 \sqrt{2}\left(a_{2}-a_{1}\right)^{-m /(m-1)} \delta^{-1 / 2}\left(\frac{\sqrt{n}}{|\alpha t|}\right)^{1 / m_{1}}
\end{aligned}
$$


и

$$
2 A<2(\sqrt{r \ln r}+1)<3 \sqrt{r \ln n}<n^{2} .
$$

Введем

и получим

$$
\theta_{m}=\frac{1}{6 m_{1}^{2} \ln m_{1}}
$$

$$
(2 A)^{\left(1-\theta_{m}\right)} \ln (2 A) \leqslant 3 r^{\left(1-\theta_{m}\right) / 2}(\ln n)^{1 / 2} \cdot 2 \log n .
$$

Следовательно, правая часть (37) не превосходит

$$
\begin{aligned}
2 \sqrt{2}(n \delta)^{-1 / 2}+\frac{18 \sqrt{2}}{\sqrt{\pi}} \widetilde{c}(m)( & n \delta)^{-\theta_{m} / 2}(\ln n)^{3 / 2} \\
& +12 \sqrt{2}\left(a_{2}-a_{1}\right)^{-m /(m-1)} \delta^{-1 / 2}\left(\frac{\sqrt{n}}{|\alpha t|}\right)^{1 / m_{1}}
\end{aligned}
$$

Напомним, что

$$
\widetilde{c}(m)>1000, \quad \theta_{m}<1 / 12, \quad n \delta>4 .
$$

Следовательно, выражение в (39) меньше, чем

$$
15 \widetilde{c}(m)(n \delta)^{-\theta_{m} / 2}(\ln n)^{3 / 2}+17\left(a_{2}-a_{1}\right)^{-m /(m-1)} \delta^{-1 / 2}\left(\frac{\sqrt{n}}{|\alpha t|}\right)^{1 / m_{1}}
$$

Условие (38) вьполнено, если

$$
0<|\alpha t| \leqslant \frac{\pi}{3 m} \cdot \frac{1}{\sqrt{\delta}} \cdot \frac{1}{\left(a_{2}-a_{1}\right)^{m}} \cdot n^{(m-1) / 2} \cdot(\ln n)^{-1 / 2},
$$

что вместе с (33) и (34) завершает доказательство теоремы 1.

\section{5. Доказательство теоремы 2}

По предположению теоремы 1 существуют $a_{1} \neq a_{2}$ такие, что

$$
\mathrm{P}\left(X_{1}=a_{1}\right)=\varepsilon_{1}>0 \text { и } \mathrm{P}\left(X_{1}=a_{2}\right)=\varepsilon_{2}>0 .
$$

Положим $\varepsilon_{3}=\min \left(\varepsilon_{1}, \varepsilon_{2}\right)$. Определим вероятностную меру $\mathrm{P}_{1}$ по формуле

$$
\mathrm{P}_{1}\left(\left\{a_{1}\right\}\right)=\mathrm{P}_{1}\left(\left\{a_{2}\right\}\right)=\frac{1}{2} .
$$

Тогда распределение $F$ случайной величины $X_{1}$ можно представить как

$$
F=2 \varepsilon_{3} \mathrm{P}_{1}+\left(1-2 \varepsilon_{3}\right) \mathrm{P}_{2}
$$

где $\mathrm{P}_{2}$ - некоторая вероятностная мера.

По теореме 1 получаем (5) для всех $n$ и $m$, бо́льших некоторых начальных значений. Для всех других возможных значений $n$ и $m$ оценка (5) вытекает из (1) при условии, что $t$ удовлетворяет (2). 


\section{6. Доказательство теоремы 3}

Пусть $U, U_{1}, U_{2}, \ldots$ - последовательность независимых одинаково распределенных случайных векторов в $\mathbb{R}^{k}$, имеюших равномерное распределение на единичном шаре $\{x:|x| \leqslant 1\}, x \in \mathbb{R}^{k}$. Используя обозначения из части $\left.3 \mathrm{~g}\right)$, запишем

$$
n_{1}=2 n_{0}, \quad Y_{n, 1} \stackrel{\mathrm{d}}{=} \eta U_{1}+a_{0} .
$$

Тогда по (33)

(40) $\sup _{a \in \mathbb{R}^{k}} \mid \mathrm{E} \exp \left\{\right.$ it $\left.f_{0}\left(S_{n}+a\right)\right\} \mid$

$$
\begin{aligned}
& \leqslant 2 \exp \left(-\frac{n_{2} \delta^{2}}{8}\right)+\sup _{a \in \mathbb{R}^{k}}\left|\mathrm{E} \exp \left\{i t f_{0}\left(\frac{\eta\left(U_{1}+\cdots+U_{n_{3}}\right)}{\sqrt{n}}+\frac{n_{3} a_{0}}{\sqrt{n}}+a\right)\right\}\right| \\
& \quad=2 \exp \left(-\frac{n_{2} \delta^{2}}{8}\right)+\sup _{a^{\prime} \in \mathbb{R}^{k}}\left|\mathrm{E} \exp \left\{i t f\left(\frac{\eta\left(U_{1}+\cdots+U_{n_{3}}\right)}{\sqrt{n}}+a^{\prime}\right)\right\}\right| .
\end{aligned}
$$

В силу (15) и (20) имеем

$$
\begin{gathered}
\sup _{a^{\prime} \in \mathbb{R}^{k}}\left|\operatorname{E} \exp \left\{i t f_{0}\left(\frac{\eta\left(U_{1}+\cdots+U_{n_{3}}\right)}{\sqrt{n}}+a^{\prime}\right)\right\}\right| \\
\leqslant 9^{k}(2 \pi)^{-k}|t|^{-k / m} \int_{\mathbb{R}^{k}}\left|g_{U}\left(\frac{\eta v}{\sqrt{n}}\right)\right|^{n_{3}} d v \\
\leqslant 9^{k}(2 \pi)^{-k} c^{*}(k)|t|^{-k / m} \eta^{-k}\left(\frac{n}{n_{3}}\right)^{k / 2}
\end{gathered}
$$

Если $n \geqslant 6 n_{0} / \delta$, то $n_{2} \geqslant n /\left(3 n_{0}\right)$ и $n_{3} \geqslant \min \left\{2, n \delta /\left(6 n_{0}\right)\right\}$. Следовательно, из (40) и (41) вытекает утверждение теоремы 3.

ЗАмечАниЕ. Мы опускаем доказательство теоремы 4, так как оно опирается на аналогичные рассуждения. Следует лишш заменить $\left|g_{U}(v)\right|$ на

$$
\left|\int_{\mathbb{R}^{k}} e^{i(v, x)} p_{0}(x) d x\right|,
$$

что, как легко видеть, меньше $(\pi /(3|v|)) V_{0}$.

\section{7. Доказательство теоремы 5}

Ясно, что, не ограничивая общности, можем предполагать, что $\alpha_{* *}=1$. Возьмем в (23)

$$
h=(2 \ln (2+|t|))^{1 / 2}>1 .
$$

Тогда первое слагаемое в правой части (23) меньше, чем

$$
k \pi^{-1 / 2}(2+|t|)^{-1} \ln ^{-1 / 2}(2+|t|) .
$$


Далее, поскольку

$$
\ln (2+|t|) \leqslant 0.125(2+|t|)^{5 / 2},
$$

имеем $2 h<(2+|t|)^{5 / 4}$ и

$$
\ln \left(2+(2 \pi)^{-1}|t|(2 h)^{M}\right)<(5 M / 4+1) \ln (2+|t|) .
$$

Следовательно, второе слагаемое в правой части (23) меньше, чем

$$
A|t|^{-1 / m} \ln ^{B}(2+|t|),
$$

где

$$
A=2^{(13 k-3 M / m) / 2}(2 \pi)^{1 / m-k / 2}(1.25 M+1)^{k-1}
$$

и $B=(3 k-M / m) / 2-1$.

Приведенные выше рассуждения доказывают теорему 5.

ЗАмечАниЕ 1 . Пусть $T$ - ортогональная $(k \times k)$-матрица и $f_{T}(x)=f(T x)$. Постоянная $m$ в (7) может быть различной для $f_{T}$ и $f$. Например, $m=1$ для $x_{1} x_{2}$, но $m=2$ для

$$
\frac{x_{1}+x_{2}}{\sqrt{2}} \cdot \frac{x_{1}-x_{2}}{\sqrt{2}}=\frac{x_{1}^{2}-x_{2}^{2}}{2} .
$$

Таким образом, подходящий выбор $T$ может улучшить оценку, даваемую теоремой 5 .

ЗАмечание 2. Можно получить результаты, аналогичные теореме 5 , заменяя $Z$ на $n^{-1 / 2}\left(U_{1}+\cdots+U_{n}\right)$. Таким путем можно доказать для общего многочлена (7) неравенство, аналогичное полученному в теореме 3 для $f_{0}$. В этом случае первый член в оценке остается без изменений, а второй имеет порядок $O\left(|t|^{-1 / m} \ln ^{b}|t|\right)$ с некоторым $b>0$ при больших значениях $|t|$.

\section{8. Доказательство теоремы 6}

Обозначим

$$
\begin{gathered}
A=v_{3}^{2} \cdots v_{k}^{2}, \quad B=v_{3}^{2}+\cdots+v_{k}^{2}, \\
D=(2 / \pi)^{(k-2) / 2}, \quad \delta=t^{-1 /(k-2)} \text { при } t \geqslant 1 .
\end{gathered}
$$

Поскольку функция $w_{k}(t)$ четная, достаточно рассмотреть лишь $t \geqslant 1$. Оценка снизу для $w_{k}(t)$ вытекает из неравенств

$$
\begin{aligned}
\mathrm{E} e^{i t f(Z)} & =\int_{0}^{\infty} \cdots \int_{0}^{\infty}\left(1+A t^{2}\right)^{-1 / 2} D e^{-B / 2} d v_{3} \ldots d v_{k} \\
& \geqslant \int_{\delta}^{1} \cdots \int_{\delta}^{1}\left(2 A t^{2}\right)^{-1 / 2} D e^{-B / 2} d v_{3} \ldots d v_{k} \\
& \geqslant 2^{-1 / 2} D e^{-(k-2) / 2} t^{-1}\left(\int_{\delta}^{1} \frac{d v}{v}\right)^{k-2}=l_{k} t^{-1} \ln ^{(k-2)} t
\end{aligned}
$$

Докажем теперь оценку сверху для $w_{k}(t)$, используя индукцию по $k$ отдельно по нечетным и четным значениям $k$. 
Из $(25)$ вытекает, что $w_{2}(t) \leqslant t^{-1}$ для всех $t>0$.

Для $t \geqslant 1$ имеем также

$$
\begin{aligned}
w_{3}(t) & =(2 \pi)^{-1 / 2} e^{1 / 4} t^{-1} K_{0}\left(\left(4 t^{2}\right)^{-1}\right) \\
& \leqslant 1.267(2 \pi)^{-1 / 2} e^{1 / 4} t^{-1} \ln \left(8 t^{2}\right)<2.6 t^{-1} \ln (2+t) .
\end{aligned}
$$

Предположим, что при некотором $k \geqslant 2$ справедливо для $t \geqslant 1$ неравенство

$$
w_{k}(t) \leqslant L_{k} t^{-1} \ln ^{k-2}(2+t) .
$$

Тогда

$$
\begin{aligned}
w_{k+2}(t) & =\frac{2}{\pi} \int_{0}^{\infty} w_{k}(t v) K_{0}(v) d v \\
& =\left(\int_{0}^{1 / t}+\int_{1 / t}^{1}+\int_{1}^{\infty}\right) \cdots=I_{1}+I_{2}+I_{3} .
\end{aligned}
$$

Из (30) находим

$$
I_{1} \leqslant 1.614 t^{-1} \ln (2+t) .
$$

Далее, по предположению индукции и (29) получаем

$$
I_{3} \leqslant \frac{2}{\pi} w_{k}(t) \int_{1}^{\infty} K_{0}(v) d v \leqslant 0.269 L_{k} t^{-1} \ln ^{k-2}(2+t) .
$$

Для $I_{2}$ имеем

$$
\begin{aligned}
I_{2} & \leqslant \frac{2}{\pi} K_{0}(1 / t) \int_{1 / t}^{1} w_{k}(t v) d v \\
& \leqslant \frac{2}{\pi} K_{0}(1 / t) L_{k} t^{-1} \int_{1 / t}^{1} v^{-1} \ln ^{k-2}(2+t v) d v \\
& <\frac{2}{\pi} K_{0}(1 / t) L_{k} t^{-1} \ln ^{k-2}(2+t) \ln t .
\end{aligned}
$$

Поскольку при $t \geqslant 1$

$$
2 / \pi K_{0}(1 / t) \leqslant 3.534 / \pi \ln (2 t)<1.075 \ln (2+t),
$$

получаем

$$
I_{2}<1.076 L_{k} t^{-1} \ln ^{k}(2+t)
$$

Таким образом, для $t \geqslant 1$

$$
w_{k+2}(t)<2.959 L_{k} t^{-1} \ln ^{k}(2+t)<L_{k+2} t^{-1} \ln ^{k}(2+t) .
$$

\section{9. Доказательство теоремы 7}

Поскольку $m_{2}$ нечетно, мы можем записать (см. следствие 3 в [23])

$$
Z_{2}^{m_{2}} \stackrel{\mathrm{d}}{=} Z_{2} S_{1}
$$


где случайные величины в правой части независимы и $S_{1} \geqslant 0$.

Имеем

$$
Z_{2}^{m_{2}} Z_{3}^{m_{3}} \cdots Z_{k}^{m_{k}} \stackrel{\mathrm{d}}{=} Z_{2} S
$$

где

$$
S \stackrel{\mathrm{d}}{=} S_{1}\left|Z_{3}\right|^{m_{3}} \ldots\left|Z_{k}\right|^{m_{k}}
$$

и

$$
Z_{1}^{m_{1}} Z_{2}^{m_{2}} \cdots Z_{k}^{m_{k}} \stackrel{\mathrm{d}}{=}\left|Z_{1}\right|^{m} Z_{2} S
$$

Объединение соотношения

$$
S^{-1 / m}\left|Z_{2}\right|^{-1 / m} \stackrel{\mathrm{d}}{=}\left|Z_{2}\right|^{-m_{2} / m} \ldots\left|Z_{k}\right|^{-m_{k} / m},
$$

(42) и леммы 8 завершает доказательство теоремы 7.

\section{СПИСОК ЛИТЕРАТУРЫ}

[1] Götze F. Asymptotic expansions for bivariate von Mises functionals // Z. Wahrscheinlichkeitstheor. verw. Geb. 1979. V. 50. № 3. P. 333-355.

[2] Götze F. Edgeworth expansions in functional limit theorems // Ann. Probab. 1989. V. 17. P. 1602-1634.

[3] Weyl H. Über die Gleichverteilung von Zahlen mod Eins // Math. Ann. 1916. V. 77. P. 313-352.

[4] Юринский В. В. Оценка погрешности нормального приближения вероятности попадания в шар // ДАН СССР. 1981. Т. 258. С. 577-578.

[5] Юринский В. В. О погрешности нормального приближения // Сиб. матем. журн. 1983. T. 24. C. $188-199$.

[6] Besicovitch A.S. Almost periodic functions. Cambridge, 1932.

[7] Cramér H. Random variables and probability distributions. Cambridge: Cambridge Univ. Press, 1970.

[8] Prachar K. Primzahlverteilung. Berlin: Springer-Verlag, 1978.

[9] Виноградов И. М. Метод тригонометрических сумм в теории чисел. М.: Наука, 1980.

[10] Rudin W. Fourier Analysis on Groups. New York: Interscience Publ., 1962.

[11] Гельфанд И. М., Райков Д. А., Шилов Г. Е. Коммутативные нормированные кольца // УМН. 1946. Т. 1. № 2 (12). С. 48-146.

[12] Uspensky J. V. Introduction to Mathematical Probability. New York: McGraw-Hill, 1937.

[13] Watson G. N. A treatise on the theory of Bessel functions. Cambridge: Cambridge Univ. Press, 1966 (Reprint of the 1944 edition).

[14] Abramowitz M., Stegun I. A. Handbook of Mathematical Functions. V. 55. Washington, D.C.: Natlional Bureau of Standards. Appl. Math., 1964.

[15] Прохоров Ю. В. Распространение неравенств С. Н. Бернштейна на многомерный случай // Теория вероятн. и ее применен. 1968. Т. 13. С. 266-274.

[16] Юринский В. В. О бесконечномерном варианте неравенств С. Н. Бернштейна // Теория вероятн. и ее применен. 1970. Т. 15. С. 106-107.

[17] Архипов Г.И., Карацуба А. А., Чубариков В.Н. Теория кратных тригонометрических сумм. М.: Наука, 1987.

[18] Cramér H. Mathematical Methods of Statistics. Princeton: Princeton Univ. Press, 1946.

[19] Садикова С. М. Некоторые неравенства для характеристических функций // Теория вероятн. и ее применен. 1966. Т. 11. С. 500-506.

[20] Bentkus V., Götze F., Zitikis R. Asymptotic expansions in the integral and local limit theorems in Banach spaces with application to $\omega$-statistics // J. Theoret. Probab. 1993. V. 6. P. $727-780$. 
[21] Багиров Э. Б. Нижние оценки для "хвостов" распределений некоторых функций // ДАН CCCP. 1990. T. 311. № 2. C. 521-523.

[22] Prudnikov A.P., Brychkov Yu. A., Marichev O. I. Integrals and series. V. 1, 2. New York: Gordon \& Breach, 1988.

[23] Багиров Э. Б. Новые следствия формулы Колмогорова для безгранично делимых законов // Теория вероятн. и ее применен. 1991. Т. 36. С. 138-143.

Билефельдский университет, ФРГ;

Поступила в редакцию

Математический институт

04.09.1995

им. В. А. Стеклова РАН, Россия;

Московский государственный

университет им. М. В. Ломоносова, Россия 